\title{
The association between varying levels of palliative care involvement on costs during terminal hospitalizations in Canada from 2012 to 2015
}

Sarina R. Isenberg ${ }^{1,2,3,4^{*}}$ D , Christopher Meaney ${ }^{2}$, Peter May ${ }^{5,6}$, Peter Tanuseputro ${ }^{1,7,8}$, Kieran Quinn ${ }^{3,9,10}$, Danial Qureshi ${ }^{1,8}$, Stephanie Saunders ${ }^{11}$, Colleen Webber ${ }^{1,8}$, Hsien Seow ${ }^{12}$, James Downar ${ }^{1,7}$, Thomas J. Smith ${ }^{13,14}$, Amna Husain ${ }^{2,11}$, Peter G. Lawlor ${ }^{1,7,8}$, Rob Fowler ${ }^{3,15,16}$, Julie Lachance ${ }^{17}$, Kimberlyn McGrail ${ }^{18}$ and Amy T. Hsu ${ }^{1,8}$

\begin{abstract}
Background: Inpatient palliative care is associated with lower inpatient costs; however, this has yet to be studied using a more nuanced, multi-tiered measure of inpatient palliative care and a national population-representative dataset. Using a population-based cohort of Canadians who died in hospital, our objectives were to: describe patients' receipt of palliative care and active interventions in their terminal hospitalization; and examine the relationship between inpatient palliative care and hospitalization costs.

Methods: Retrospective cohort study using data from the Discharge Abstract Database in Canada between fiscal years 2012 and 2015. The cohort were Canadian adults (age $\geq 18$ years) who died in hospital between April 1st, 2012 and March 31st, $2015(N=250,640)$. The exposure was level of palliative care involvement defined as: mediumhigh, low, or no palliative care. The main measure was acute care costs calculated using resource intensity weights multiplied by the cost of standard hospital stay, represented in 2014 Canadian dollars (CAD). Descriptive statistics were represented as median (IQR), and $\mathrm{n}(\%)$. We modelled cost as a function of palliative care using a gamma generalized estimating equation (GEE) model, accounting for clustering by hospital.

Results: There were 250,640 adults who died in hospital. Mean age was 76 (SD 14), 47\% were female. The most common comorbidities were: metastatic cancer (21\%), heart failure (21\%), and chronic obstructive pulmonary disease (16\%). Of the decedents, 95,450 (38\%) had no palliative care involvement, 98,849 (38\%) received low involvement, and 60,341 (24\%) received medium to high involvement. Controlling for age, sex, province and predicted hospital mortality risk at admission, the cost per day of a terminal hospitalization was: $\$ 1359(95 \% \mathrm{Cl}$ 1323: 1397) (no involvement), \$1175 (95\% Cl 1146: 1206) (low involvement), and \$744 (95\% Cl 728: 760) (mediumhigh involvement).

(Continued on next page)
\end{abstract}

\footnotetext{
* Correspondence: sisenberg@bruyere.org

'Bruyère Research Institute, 43 Bruyère St, Office 264J-G, Ottawa, ON K1N 5C8, Canada

${ }^{2}$ Department of Family and Community Medicine, University of Toronto, Toronto, Canada

Full list of author information is available at the end of the article
}

(c) The Author(s). 2021 Open Access This article is licensed under a Creative Commons Attribution 4.0 International License, which permits use, sharing, adaptation, distribution and reproduction in any medium or format, as long as you give appropriate credit to the original author(s) and the source, provide a link to the Creative Commons licence, and indicate if changes were made. The images or other third party material in this article are included in the article's Creative Commons licence, unless indicated otherwise in a credit line to the material. If material is not included in the article's Creative Commons licence and your intended use is not permitted by statutory regulation or exceeds the permitted use, you will need to obtain permission directly from the copyright holder. To view a copy of this licence, visit http://creativecommons.org/licenses/by/4.0/ The Creative Commons Public Domain Dedication waiver (http://creativecommons.org/publicdomain/zero/1.0/) applies to the data made available in this article, unless otherwise stated in a credit line to the data. 
(Continued from previous page)

Conclusions: Increased involvement of palliative care was associated with lower costs. Future research should explore whether this relationship holds for non-terminal hospitalizations, and whether palliative care in other settings impacts inpatient costs.

Keywords: Palliative care, Acute care costs, Terminal hospitalizations, End of life

\section{Background}

High proportions of deaths in hospitals are an issue confronting several countries. In 2012 in Belgium, Canada, England, Germany, and Norway, the proportion of decedents who died in acute care hospitals ranged from 42 to $52 \%$ [1]. In $2018,59.5 \%$ of all deaths in Canada occurred in hospital [2]. These terminal hospitalizations (i.e., hospitalizations wherein a patient dies) are costly. According to a study of Ontario decedents, $\$ 4.7$ billion CAD was spent on health care in the last year of life and $42.9 \%$ of costs are derived from inpatient costs (an average of $\$ 30,872 \mathrm{CAD}$ per person) [3]. Focusing on the last 180 days of life for patients with cancer, Canada had the highest per capita hospital expenditures at \$21,840 USD, compared to the United States, Norway, Germany, Belgium, and the Netherlands [1].

Treatments that tend to drive these end-of-life costs are sometimes termed aggressive interventions, such as admission to intensive care units (ICU), mechanical ventilation, defibrillation, dialysis, percutaneous coronary interventions, and percutaneous feeding tube insertions. A recent study of decedents in Ontario demonstrated that, in the last 6 months of life, $19.4 \%$ were admitted to the ICU, and $13.9 \%$ received mechanical ventilation [4].

A meta-analysis on the association between palliative care consultations and direct hospital costs for adults with serious illness found that patients who received palliative care consultations had a statistically significant reduction in direct hospital costs (-\$3237 USD; 95\% CI, -\$3581 to $-\$ 2893 ; P<.001)$ [5]. There are limitations to previous studies. Most studies focus on individual hospital or state-level costs. Most previous studies analyzed the relationship between palliative care and costs as a binary variable [6-9]. In reality there are differing levels of inpatient palliative care involvement. A binary variable does not give a good sense of how much palliative care people are receiving, and a more nuanced variable can help decision makers determine how to appropriately allocate resources.

Due to past studies' lack of nuanced palliative care variables and lack of national scope, we sought to understand the relationship between differing levels of inpatient palliative care and hospitalization costs in terminal hospitalizations. Our first objective was to describe patients who died in hospital, and their receipt of different levels of palliative care and active interventions.
Our second objective was to examine the relationship between terminal hospitalizations with varying levels of palliative care involvement and costs.

\section{Methods \\ Study design, setting, and data sources}

We conducted a national retrospective cohort study, using patient-level health administrative data from the Discharge Abstract Database (DAD) in Canada (excluding the province of Quebec ${ }^{1}$ and the territories). The DAD is a national database that includes data from acute care institutions across Canada. It is mandatory for all provinces, except Quebec, to submit a DAD abstract for each hospital discharge. A trained Canadian Institute for Health Information data abstractor reviews the medical record for the encounter and makes determinations about the care received to populate the DAD. Analysis was conducted from January 2020-July 2020.

\section{Study cohort}

Our cohort included all adults who died in hospital between April 1st, 2012 and March 31st, 2015. We excluded people who were under age 18 or over age 105 at death, or who were non-Canadian residents. We excluded people with sex not identified as either male or female because there were few records and we could not meaningfully analyze the end-of-life experience of these individuals. We excluded people with missing postal code data, missing admission/discharge dates, or missing/zero inpatient resource intensity weight data, which results in missing/zero cost data. We excluded people from Yukon, Northwest Territories, and Nunavut due to small sample sizes and data unavailable for costs of a standard hospital stay in the Northwest Territories and Nunavut. We also excluded people with erroneous data entries concerning their admissions and death (e.g., multiple terminal admissions). Finally, we excluded people whose terminal hospitalization had a length of stay greater than 180 days, as these people are not representative of a typical acute care stay and may be capturing long-term care beds that exist within some Canadian hospitals.

\footnotetext{
${ }^{1}$ Note: Quebec does not contribute to the DAD data collection.
} 


\section{Patient characteristics}

We measured demographic and clinical variables including age, sex, rurality, province, comorbidities and chronic conditions defined as per the Elixhauser index [10] and the Van Walraven index (a weighted numeric score based on the Elixhauser classification system designed to predict in-hospital mortality) [11], represented as empirical quartiles of the score observed in our sample. We also measured ICU stay, receipt of major surgery [12] and "any active intervention", defined as mechanical ventilation, cardiopulmonary resuscitation (CPR), defibrillation, dialysis, percutaneous coronary intervention, percutaneous feeding tube insertion, blood transfusion, and bronchoscopy. We developed this list of active interventions based on consensus among our group of experts in palliative and intensive care.

\section{Exposure}

Our primary exposure was palliative care involvement, adapted from Webber et al. [13]. For each admission, the DAD captures up to 25 diagnosis codes based on the 10th revision of the International Statistical Classification of Diseases and Related Health Problems (ICD-10). Medium-high palliative involvement was defined as a hospitalization with palliative care as the most responsible service provider (i.e., the service that treated the patient for the majority of their hospital stay; this could include care delivered by a specialist palliative care provider or a generalist providing palliative care) and/or the most responsible diagnosis (i.e., when the patient is admitted solely to receive palliative care or when the patient received palliative care prior to hospital arrival but was not admitted for a reversible cause; using ICD-10 diagnosis code Z51.5). Low Palliative involvement was defined as a hospitalization with a secondary diagnosis of palliative care (i.e., using diagnosis codes 2 through $25)$, excluding most responsible provider. No involvement was defined as a hospitalization without a palliative diagnosis or most responsible service provider. In Canada, inpatient palliative care is largely provided as a consult service; here palliative care would not be noted as most responsible provider, but would likely be noted as most responsible diagnosis or secondary diagnosis. Inpatient palliative care might also include palliative care units, which would have the palliative care designation as most responsible provider.

\section{Outcome}

The total costs of a terminal hospitalization were calculated using validated costing methods [14-17]. Each acute care hospital admission is associated with an inpatient resource intensity weight (RIW), which measures the intensity of resource use associated with medical, diagnostic and surgical procedures, adjusted for patient and institutional characteristics (e.g., case-mix adjustments). Inpatient hospital costs are calculated by multiplying the RIWs by a cost-of-standard-hospital-stay, which is measured at the provincial level. We also adjusted costs to 2014 using the Statistics Canada Health Care Consumer Price Index (CPI) [18]. All costs are in Canadian dollars.

\section{Statistical analysis}

For Objective 1, wherein we describe patients who died in hospital and their receipt of palliative care, we presented descriptive statistics as mean (standard deviation $(\mathrm{SD})$ ), median (interquartile range (IQR)), and $\mathrm{n}(\%)$. For Objective 2, wherein we examined the relationship between varying levels of palliative care involvement and costs, we fit a generalized estimating equation (GEE) model, assuming our response variable (inpatient hospital costs) followed a gamma distribution. We used a log link function. We accounted for clustering by hospital using a compound symmetric working correlation structure. We include the natural logarithm of patient length of stay as an offset in our gamma GEE models. Our unadjusted model had palliative care involvement as the only predictor and costs as the outcome. Based on clinical expertise and estimated bivariate associations from our unadjusted models, we built a multivariable gamma GEE model controlling for age, sex, province, and Van Walraven index. We present regression coefficient estimates (representing log cost-ratios), 95\% confidence intervals (CIs) and $p$-values from the fitted gamma GEE models. For both unadjusted and adjusted models, we present the conditional expected value of cost (and its 95\% CI) at each level of palliative care intensity, controlling for other variables in the model.

We conducted two sensitivity analyses. First, we estimated stratified multivariable models by province because palliative care delivery [19] and the cost of a standard hospital stay differs across provinces. Second, we estimated stratified multivariable models by Elixhauser comorbidity categories [10], specifically metastatic cancer, heart failure, major neurocognitive disorders, chronic obstructive pulmonary disease (COPD), kidney failure, human immunodeficiency virus/acquired immunodeficiency syndrome (HIV/AIDS), and liver failure. We focused on these diseases categories as they are known to play a role in the relationship between palliative care receipt and associated costs [5, 20]. We report adjusted costs from the stratified gamma GEE model fits. All analyses were performed using SAS version 9.4 (SAS Institute, Cary, North Carolina).

This study was approved by Institutional Ethics Review Boards at Sinai Health and the Ottawa Health Services Network. While data sharing agreements prohibit the research team and Canadian Institute for Health 
Table 1 Patient demographic and clinical characteristics stratified by level of palliative care involvement

\begin{tabular}{|c|c|c|c|c|}
\hline Category & $\begin{array}{l}n \\
(n=250,640)\end{array}$ & $\begin{array}{l}\text { No involvement } n(\%) \\
(n=95,450)\end{array}$ & $\begin{array}{l}\text { Low involvement } n(\%) \\
(n=98,849)\end{array}$ & $\begin{array}{l}\text { Medium-high involvement } \\
\mathrm{n}(\%)(n=60,341)\end{array}$ \\
\hline \multicolumn{5}{|l|}{ Age } \\
\hline $18-40$ & 4401 & $2280(2)$ & $1382(2)$ & $739(1)$ \\
\hline $41-55$ & 16,778 & $6157(6)$ & $5948(6)$ & $4673(8)$ \\
\hline $56-70$ & 54,974 & $18,999(20)$ & $20,248(21)$ & $15,727(26)$ \\
\hline $71-85$ & 104,250 & $39,738(42)$ & $40,139(42)$ & $24,373(40)$ \\
\hline 86-105 & 70,237 & $28,276(30)$ & $27,132(29)$ & $14,829(25)$ \\
\hline \multicolumn{5}{|l|}{ Sex } \\
\hline $\mathrm{F}$ & 118,534 & $43,364(45)$ & $45,211(48)$ & $29,959(50)$ \\
\hline \multicolumn{5}{|l|}{ Rurality } \\
\hline Yes & 49,563 & $20,286(21)$ & 15,965 (17) & $13,312(22)$ \\
\hline \multicolumn{5}{|l|}{ Province } \\
\hline$A B$ & 29,562 & $12,591(13)$ & $10,869(11)$ & $6102(10)$ \\
\hline$B C$ & 41,283 & $16,333(17)$ & $16,103(17)$ & 8847 (15) \\
\hline $\mathrm{MB}$ & 15,244 & 4776 (5) & $5654(6)$ & $4814(8)$ \\
\hline NB & 10,992 & 3435 (4) & $3920(4)$ & $3637(6)$ \\
\hline $\mathrm{NL}$ & 7199 & 2499 (3) & 2388 (3) & $2312(4)$ \\
\hline NS & 13,494 & 3271 (3) & $4936(5)$ & $5287(9)$ \\
\hline ON & 118,410 & $46,639(49)$ & $46,200(49)$ & $25,571(42)$ \\
\hline$P E$ & 1856 & $675(1)$ & $696(1)$ & $485(1)$ \\
\hline SK & 12,600 & $5231(5)$ & $4083(4)$ & $3286(5)$ \\
\hline \multicolumn{5}{|l|}{ Number of comorbidities } \\
\hline 0 & 25,186 & $12,807(13)$ & $8176(9)$ & $4203(7)$ \\
\hline 1 & 84,879 & $26,569(28)$ & $28,184(30)$ & $30,126(50)$ \\
\hline 2 & 64,604 & $24,015(25)$ & $25,842(27)$ & $14,747(24)$ \\
\hline $3+$ & 75,971 & $32,059(34)$ & $32,647(34)$ & $11,265(19)$ \\
\hline \multicolumn{5}{|l|}{ Hospital length of stay (days) } \\
\hline $0-2$ & 56,892 & $28,992(30)$ & $13,294(14)$ & $14,606(24)$ \\
\hline $3-7$ & 64,081 & $25,527(27)$ & $22,905(24)$ & $15,649(26)$ \\
\hline $8-19$ & 66,797 & $21,987(23)$ & $29,571(31)$ & $15,239(25)$ \\
\hline $20+$ & 62,870 & $18,944(20)$ & $29,079(31)$ & $14,847(25)$ \\
\hline Heart failure & 52,510 & $25,076(26)$ & $21,711(23)$ & $5723(9)$ \\
\hline COPD & 39,731 & $17,240(18)$ & $16,541(17)$ & $5950(10)$ \\
\hline HIV & 418 & $181(0)$ & $170(0)$ & $67(0)$ \\
\hline Liver failure & 16,096 & $6295(7)$ & $7457(8)$ & $2344(4)$ \\
\hline Metastatic cancer & 52,734 & $6992(7)$ & $19,475(21)$ & $26,267(44)$ \\
\hline Major neurocognitive disorders & 19,249 & $7393(8)$ & $8545(9)$ & $3311(5)$ \\
\hline Kidney failure & 23,130 & $10,042(11)$ & $9749(10)$ & $3339(6)$ \\
\hline \multicolumn{5}{|l|}{ Van Walraven Index } \\
\hline Quartile -1-3 & 58,870 & $28,693(30)$ & $19,747(21)$ & $10,430(17)$ \\
\hline Quartile 4-8 & 65,252 & $27,308(29)$ & $23,490(25)$ & $14,454(24)$ \\
\hline Quartile 9-15 & 60,678 & $23,967(25)$ & $24,750(26)$ & $11,961(20)$ \\
\hline Quartile 16+ & 65,840 & $15,482(16)$ & $26,862(28)$ & $23,496(39)$ \\
\hline
\end{tabular}


Table 1 Patient demographic and clinical characteristics stratified by level of palliative care involvement (Continued)

\begin{tabular}{|c|c|c|c|c|}
\hline Category & $\begin{array}{l}n \\
(n=250,640)\end{array}$ & $\begin{array}{l}\text { No involvement } n(\%) \\
(n=95,450)\end{array}$ & $\begin{array}{l}\text { Low involvement } n(\%) \\
(n=98,849)\end{array}$ & $\begin{array}{l}\text { Medium-high involvement } \\
\mathrm{n}(\%)(n=60,341)\end{array}$ \\
\hline Major Surgery ${ }^{a}$ & 47,068 & $23,218(24)$ & $20,825(22)$ & $3025(5)$ \\
\hline ICU stay & 75,302 & $39,774(42)$ & $31,117(33)$ & $4411(7)$ \\
\hline Mechanical ventilation & 52,022 & $29,487(31)$ & $20,288(21)$ & $2247(4)$ \\
\hline CPR & 13,656 & $10,920(11)$ & $2551(3)$ & $185(0)$ \\
\hline Defibrillation & 5172 & $3789(4)$ & $1293(1)$ & $90(0)$ \\
\hline Dialysis & 11,860 & $6102(6)$ & $5179(5)$ & $579(1)$ \\
\hline Percutaneous Coronary Intervention & 2616 & $1865(2)$ & $690(1)$ & $61(0)$ \\
\hline Feeding tube & 6626 & $2956(3)$ & $2993(3)$ & $677(1)$ \\
\hline Blood Transfusion & 201 & $96(0)$ & $81(0)$ & $24(0)$ \\
\hline Bronchoscopy & 3264 & $1633(2)$ & $1469(2)$ & $162(0)$ \\
\hline Any active intervention ${ }^{\mathrm{b}}$ & 64,897 & $36,726(38)$ & $24,729(26)$ & $3442(6)$ \\
\hline
\end{tabular}

Abbreviations: F Female, AB Alberta, BC British Columbia, MB Manitoba, NB New Brunswick, NL Newfoundland, NS Nova Scotia, ON Ontario, PE Prince Edward Island, SK Saskatchewan, COPD Chronic obstructive pulmonary disease, HIV Human immunodeficiency virus, ICU Intensive care unit, CPR Cardiopulmonary resuscitation ${ }^{a}$ Major surgery is defined according to anatomic site: abdominal, cardiac, retroperitoneal, thoracic and vascular [12]

${ }^{b}$ Any active intervention is defined as any of the following: mechanical ventilation, CPR, defibrillation, dialysis, percutaneous coronary intervention, feeding tube, blood transfusion, and bronchoscopy

Information from making the dataset publicly available, access may be granted to those who meet pre-specified criteria for confidential access. The underlying analytic code are available from the authors upon request, with the understanding that the programs may rely on coding templates or macros that are unique to Canadian Institute for Health Information.

\section{Results}

\section{Baseline characteristics}

We identified 290,855 adult hospital decedents. We excluded 40,215 adults, resulting in a cohort of 250,640 people (see Supplementary Appendix for a full list of exclusions). Of the decedents, 95,450 (38\%) had no inpatient palliative care involvement, 98,849 (38\%) received low palliative care involvement, and 60,341 (24\%) received medium-high palliative care involvement. Mean age was 76 years ( $\mathrm{SD}=14$ years), $47 \%$ were female, $47 \%$ were from Ontario, and $26 \%$ received any active intervention (Table 1). The most common comorbidities were: metastatic cancer (21\%), heart failure $(21 \%)$, and COPD (16\%). Table 2 displays the terminal hospitalization episode costs per day according to patient characteristics. Costs decreased as level of palliative care involvement increased. There was large provincial variation in mean cost of terminal hospitalization episode with the highest median costs per day being in Alberta at $\$ 1250$ (IQR 1022-1684) and the lowest median costs being in New Brunswick at $\$ 797$ (IQR 657-1015). Median costs were higher among patients who received any active intervention at $\$ 2088$ (IQR 1396-2981) compared to those who received no active intervention at $\$ 881$ (IQR 730-1074). The Supplementary Appendix displays a table demonstrating the relationship between level of palliative care involvement, daily costs, and length of stay; as level of palliative care involvement increases, cost per day decreases, and as length of stay increases, costs decrease.

\section{Main analyses}

In the unadjusted analysis, compared to no palliative care involvement, palliative care involvement was associated with lower costs per hospital-day (Table 3). At no involvement (i.e., the reference), the mean cost per hospital-day was \$1414 (95\% CI 1377: 1453). At low involvement, the cost was $\$ 1180$ (95\% CI 1155: 1206). At medium-high involvement, the cost was $\$ 737$ (95\% CI 721: 753).

After adjusting for age, sex, province, and Van Walraven index, increasing levels of palliative care involvement were incrementally associated with lower costs, compared to no palliative care involvement (Table 3). Among decedents with no involvement (i.e., the reference), the cost per hospital day was $\$ 1359$ (95\% CI 1323: 1397). At low involvement, the cost was $\$ 1175$ (95\% CI 1146: 1206). At medium-high involvement, the cost was \$744 (95\% CI 728: 760).

\section{Sensitivity analyses}

Adjusting for age, sex, and Van Walraven index, we found that higher palliative care involvement was associated with lower costs in all provinces (Table 4) Adjusting for age, sex, province, and Van Walraven index, we found that higher palliative care involvement was 
Table 2 Costs per day of a terminal hospitalizations according to patient demographic and clinical characteristics

\begin{tabular}{|c|c|c|c|c|c|}
\hline Category & $\mathbf{n}$ & Costs median (IQR) & Category & $\mathbf{n}$ & Costs median (IQR) \\
\hline Palliative care involvement & & & Metastatic cancer & & \\
\hline No involvement & 95,450 & $1154(888-1882)$ & No & 197,906 & $1037(824-1588)$ \\
\hline Low involvement & 94,849 & $1032(856-1486)$ & Yes & 52,734 & 815 (694-1033) \\
\hline Medium-high involvement & 60,341 & $726(661-875)$ & Major neurocognitive disorders & & \\
\hline Age & & & No & 231,391 & $967(775-1384)$ \\
\hline $18-40$ & 4401 & $1834(1040-2974)$ & Yes & 19,249 & $1207(857-2247)$ \\
\hline $41-55$ & 16,778 & $1186(818-2221)$ & Kidney failure & & \\
\hline $56-70$ & 54,974 & $1050(779-1803)$ & No & 227,510 & $972(773-1435)$ \\
\hline $71-85$ & 104,250 & 981 (783-1419) & Yes & 23,130 & $1031(845-1440)$ \\
\hline $85+$ & 70,237 & $911(768-1139)$ & Van Walraven Index & & \\
\hline Sex & & & Quartile -14-3 & 58,870 & $1047(816-1636)$ \\
\hline $\mathrm{F}$ & 118,534 & $957(771-1352)$ & Quartile 4-8 & 65,252 & $991(786-1496)$ \\
\hline M & 132,106 & $1001(790-1520)$ & Quartile 9-15 & 60,678 & 1010 (819-1473) \\
\hline Rurality & & & Quartile 16+ & 65,840 & 897 (726-1197) \\
\hline Non-rural & 201,077 & $985(781-1464)$ & Hospital Length of Stay & & \\
\hline Rural & 49,563 & 955 (774-1317) & $0-3$ & 56,892 & $1062(745-1643)$ \\
\hline Province & & & $4-7$ & 64,081 & 985 (823-1371) \\
\hline$A B$ & 29,562 & $1250(1022-1684)$ & $8-20$ & 66,797 & 972 (805-1389) \\
\hline$B C$ & 41,283 & 947 (770-1303) & $30+$ & 62,870 & $929(747-1325)$ \\
\hline MB & 15,244 & $911(748-1225)$ & ICU stay & & \\
\hline NB & 10,992 & 797 (657-1015) & No & 175,338 & $877(727-1077)$ \\
\hline $\mathrm{NL}$ & 7199 & $957(774-1268)$ & Yes & 75,302 & $1808(1146-2788)$ \\
\hline NS & 13,494 & 877 (718-1145) & Mechanical ventilation & & \\
\hline ON & 118,410 & $949(761-1481)$ & No & 198,618 & $895(740-1103)$ \\
\hline PE & 1856 & $919(757-1161)$ & Yes & 52,022 & $2350(1647-3151)$ \\
\hline SK & 12,600 & $1103(897-1504)$ & CPR & & \\
\hline Number of comorbidities & & & No & 236,984 & $956(770-1343)$ \\
\hline 0 & 25,186 & $1073(823-1784)$ & Yes & 13,656 & 2009 (1366-2926) \\
\hline 1 & 84,879 & $894(717-1228)$ & Defibrillation & & \\
\hline 2 & 64,604 & 975 (787-1408) & No & 245,468 & 969 (776-1394) \\
\hline $3+$ & 75,971 & $1054(848-1582)$ & Yes & 5172 & $2310(1508-3352)$ \\
\hline Heart failure & & & Dialysis & & \\
\hline No & 198,130 & $961(758-1433)$ & No & 238,780 & $960(771-1359)$ \\
\hline Yes & 52,510 & $1031(863-1446)$ & Yes & 11,860 & $2035(1294-3042)$ \\
\hline COPD & & & Percutaneous Coronary Interven & & \\
\hline No & 210,909 & 966 (769-1433) & No & 248,024 & $973(778-1409)$ \\
\hline Yes & 39,731 & 1024 (849-1444) & Yes & 2616 & $3611(2560-5087)$ \\
\hline HIV & & & Feeding tube & & \\
\hline No & 250,222 & $977(780-1434)$ & No & 244,014 & $969(775-1402)$ \\
\hline Yes & 418 & $1440(1015-2344)$ & Yes & 6626 & $1609(1114-2577)$ \\
\hline Liver failure & & & Blood transfusion & & \\
\hline No & 234,544 & $966(774-1393)$ & No & 250,439 & $978(780-1435)$ \\
\hline Yes & 16,096 & $1220(907-2091)$ & Yes & 201 & $1580(1004-2949)$ \\
\hline
\end{tabular}

Table 2 Costs per day of a terminal hospitalizations according to patient demographic and clinical characteristics (Continued) 
Table 2 Costs per day of a terminal hospitalizations according to patient demographic and clinical characteristics (Continued)

\begin{tabular}{lll}
\hline Category & $\mathbf{n}$ & Costs median (IQR) \\
\hline $\begin{array}{lll}\text { Bronchoscopy } \\
\text { No }\end{array}$ & 247,376 & $972(777-1406)$ \\
Yes & 3264 & $2604(1757-3206)$ \\
Any active intervention ${ }^{\mathrm{a}}$ & & \\
No & 185,743 & $881(730-1074)$ \\
Yes & 64,897 & $2088(1396-2981)$ \\
Major surgery & & \\
No & & \\
Yes & 203,572 & $902(744-1134)$ \\
\hline Abbrations & 47,068 & $2046(1435-2999)$ \\
\hline
\end{tabular}

Abbreviations: $F$ Female, $M$ Male, $A B$ Alberta, $B C$ British Columbia, $M B$ Manitoba, $N B$ New Brunswick, NL Newfoundland, NS Nova Scotia, ON Ontario, PE Prince Edward Island, SK Saskatchewan, COPD Chronic obstructive pulmonary disease, HIV Human immunodeficiency virus, ICU Intensive care unit, CPR

Cardiopulmonary resuscitation

${ }^{a}$ Any active intervention is defined as any of the following: mechanical ventilation, CPR, defibrillation, dialysis, percutaneous coronary intervention, feeding tube, blood transfusion, and bronchoscopy

${ }^{\mathrm{b}}$ Major surgery is defined according to anatomic site: abdominal, cardiac, retroperitoneal, thoracic and vascular [12]

associated with lower costs across Elixhauser comorbidity categories (Table 5).

\section{Discussion}

In this study, we demonstrated increased involvement of palliative care was associated with lower costs.. However, a substantial proportion (38\%) had no inpatient palliative care involvement in their terminal hospitalization and a minority (24\%) received medium-high palliative care involvement. In addition to cost, these findings are also reflective of the care experience of Canadians who die in hospitals; our analysis found that the proportion of decedents with any active interventions decreased with increasing levels of palliative care involvement. This relationship between palliative care involvement and costs was sustained while stratifying across provinces and disease groups.

The relatively low level of inpatient palliative care receipt was also observed in a national study of decedents who were hospitalized in their last year of life, wherein $18.7 \%$ received inpatient palliative care consultation [21]. In our study, the majority of people received little to no palliative care in their terminal hospitalization. This finding is in line with our past work on terminal hospitalizations in Ontario from 2012 to 2015, which found that $36 \%$ never had access to palliative care [22]. These low levels of palliative care receipt are problematic, as the Canadian Institutes for Health Information estimates that $94 \%$ of patients who died in hospital were hospitalized for a non-sudden death condition that could potentially have benefitted from palliative care involvement [19]; however, it is unclear how that report operationalizes potential benefit.
Our findings reinforce past studies demonstrating that inpatient palliative care is associated with lower inpatient costs $[5-9,23]$. A systematic review of the economic evidence on specialist palliative care consultations found that there were statistically significant savings of $9-25 \%$ associated with palliative care consultation [23]. However, ours is the first national study to use a tiered palliative care variable demonstrating the relationship between palliative care and lower costs. Importantly, our findings are restricted to terminal hospitalizations and future studies would need to analyze whether this relationship holds for other hospitalizations.

These lower costs associated with palliative care might be a consequence of patients receiving palliative care undergoing fewer active interventions and having fewer ICU stays, which is likely aligned with the patients' underlying preferences for care. Other studies have found that palliative care consultations are associated with lower intensity of hospital treatment, less expensive procedures, shorter length of stay [23-30], decreased use of chemotherapy near death, and lower risk of ICU admission, multiple emergency department visits, and multiple hospitalizations near death [31]. Hsu et al.'s national, Canadian study found that, in an adjusted analysis, receipt of palliative care was associated with lower odds of ICU admission in the last 30 days of life (OR: 0.31, 95\% CI: 0.30 0.32) [21]. Importantly, while palliative care is associated with a reduced likelihood of receipt of these treatments, it may be because patients' preferences to receive less aggressive care is driving higher rates of palliative care and lower rates of these active interventions. Further, lifesustaining measures can cause or prolong suffering in a dying person, but the goals of palliative care and intensive care are not mutually exclusive. There are many models for integrating palliative care principles into the critical care environment [32], and one recent study found that among hospital decedents, ICU care was associated with higher family ratings of quality of end-of-life care than ward care [33].

The dose-response relationship between palliative care and costs was also observed across disease groups, including metastatic cancer, heart failure, major neurocognitive disorders, COPD, kidney failure, HIV/AIDS, and liver failure. These findings are consistent with diseasespecific palliative care economic evaluations. A metaanalysis of six studies on the economic impact of inpatient palliative care consultations, found that for studies focusing on cancer, palliative care led to a reduction in costs (-\$4251 USD; 95\% CI, $-\$ 4664$ to $-\$ 3837$ ). The same study found that for noncancer populations, there was also an association between palliative care and lower costs ( $\$ 2105 ; 95 \% \mathrm{CI},-\$ 2698$ to $-\$ 1511)$ [5]. May et al. conducted a pooled analysis of four economic evaluations of non-cancer diseases. They found that 
Table 3 Regression coefficient estimates (on log cost-ratio scale ${ }^{\mathrm{a}}$ ), 95\% confidence intervals and $p$-values from unadjusted and adjusted gamma Generalized Estimating Equation models for cost per day outcome

\begin{tabular}{|c|c|c|c|c|}
\hline \multirow[t]{2}{*}{ Category } & \multicolumn{2}{|l|}{ Unadjusted Models ${ }^{\mathbf{b}}$} & \multicolumn{2}{|l|}{ Adjusted Model $^{c}$} \\
\hline & Coefficient $(95 \% \mathrm{Cl})$ & $P$-value & Coefficient $(95 \% \mathrm{Cl})$ & $P$-value \\
\hline \multicolumn{5}{|l|}{ Palliative Care Intensity } \\
\hline No involvement & Ref & & Ref & \\
\hline Low involvement & $-0.18(-0.21:-0.15)$ & $<0.001$ & $-0.15(-0.17:-0.12)$ & $<0.001$ \\
\hline Medium involvement & $-0.65(-0.69:-0.62)$ & $<0.001$ & $-0.6(-0.63:-0.57)$ & $<0.001$ \\
\hline \multicolumn{5}{|l|}{ Age } \\
\hline $18-40$ & Ref & & Ref & \\
\hline $41-55$ & $-0.22(-0.27:-0.18)$ & $<0.001$ & $-0.15(-0.18:-0.11)$ & $<0.001$ \\
\hline $56-70$ & $-0.32(-0.36:-0.27)$ & $<0.001$ & $-0.23(-0.27:-0.19)$ & $<0.001$ \\
\hline $71-85$ & $-0.45(-0.5:-0.39)$ & $<0.001$ & $-0.38(-0.42:-0.34)$ & $<0.001$ \\
\hline $86-104$ & $-0.64(-0.7:-0.59)$ & $<0.001$ & $-0.58(-0.62:-0.54)$ & $<0.001$ \\
\hline \multicolumn{5}{|l|}{ Sex } \\
\hline $\mathrm{F}$ & Ref & & Ref & \\
\hline M & 0.08 (0.07: 0.09) & $<0.001$ & 0.04 (0.03: 0.05) & $<0.001$ \\
\hline \multicolumn{5}{|l|}{ Province } \\
\hline ON & Ref & & Ref & \\
\hline$A B$ & $-0.02(-0.19: 0.14)$ & 0.79 & $0.02(-0.07: 0.11)$ & 0.66 \\
\hline$B C$ & $0.02(-0.1: 0.13)$ & 0.78 & $0.03(-0.05: 0.11)$ & 0.48 \\
\hline $\mathrm{MB}$ & $-0.02(-0.14: 0.11)$ & 0.81 & 0 (-0.08: 0.09) & 0.96 \\
\hline NB & $-0.02(-0.35: 0.31)$ & 0.91 & $-0.01(-0.24: 0.21)$ & 0.9 \\
\hline $\mathrm{NL}$ & $0.05(-0.1: 0.2)$ & 0.52 & 0.04 (-0.06: 0.13) & 0.44 \\
\hline NS & $-0.19(-0.61: 0.24)$ & 0.39 & $-0.11(-0.37: 0.15)$ & 0.4 \\
\hline PE & $0.48(0.17: 0.8)$ & 0.002 & 0.39 (0.09: 0.69) & 0.01 \\
\hline SK & $0.06(-0.03: 0.14)$ & 0.23 & 0.07 (0.01: 0.13) & 0.02 \\
\hline \multicolumn{5}{|l|}{ Van Walraven Index } \\
\hline$-14-3$ & Ref & & Ref & \\
\hline $4-8$ & $-0.07(-0.08:-0.05)$ & $<0.001$ & $-0.04(-0.05:-0.03)$ & $<0.001$ \\
\hline $9-15$ & $-0.1(-0.12:-0.08)$ & $<0.001$ & $-0.09(-0.1:-0.07)$ & $<0.001$ \\
\hline $16+$ & $-0.25(-0.28:-0.22)$ & $<0.001$ & $-0.2(-0.22:-0.17)$ & $<0.001$ \\
\hline
\end{tabular}

Abbreviations: F Female, $A B$ Alberta, BC British Columbia, MB Manitoba, NB New Brunswick, NL Newfoundland, NS Nova Scotia, ON Ontario, PE Prince Edward Island, SK Saskatchewan

${ }^{a}$ As explained in previous work, these "coefficient estimates [are] derived from generalized linear models with logarithmic link functions [and] are interpretable as the logarithm of the relative change in mean cost associated with a one-unit change in the predictor variable" (Austin PC, Ghali WA, Tu JV. A comparison of several regression models for analysing cost of CABG surgery. Stat Med. 2003 Sep 15;22 (17):2799-815. doi: https://doi.org/10.1002/sim.1442. PMID: 12939787) ${ }^{\mathrm{b}}$ This column displays unadjusted models for each variable

${ }^{c}$ Model adjusted for age, sex, province, and Van Walraven index

inpatient palliative care was associated with a reduction in total direct costs for heart failure, neurodegenerative conditions, COPD, kidney failure, and liver failure. They did not find an association for HIV/AIDS [20].

The magnitude of costs varied across provinces, which is expected, as there are differences in provincial offerings of palliative care, and cost-of-standard-hospital-stay. However, some of these observed differences might be due to inter-provincial administrative coding differences. The mandatory elements to be coded vary by province, and there are noted inter-provincial inconsistencies in the format and coding of data elements, including diagnoses and procedures [34]. Notably, in our descriptive findings, the mean cost per day in Alberta was higher than any other province at $\$ 1250$. This may relate to two factors, though further analysis is required to determine a causal link. First, Alberta has the highest per capita health spending of all the provinces, estimated at $\$ 7658$ per person in 2019 , compared to the national average at $\$ 7068$ [35]. Second, Alberta has the lowest levels of inpatient palliative care of all the provinces at $15.2 \%$ [21]. When comparing people who died in acute care from 2016 to 2017, Alberta 
Table 4 Estimated costs per day and 95\% confidence intervals from adjusted ${ }^{a}$ gamma Generalized Estimating Equation (GEE) model, stratified by province

\begin{tabular}{|c|c|c|c|}
\hline Province & Palliative care involvement & $\mathrm{n}$ & Costs $(95 \% \mathrm{Cl})$ \\
\hline \multirow[t]{3}{*}{ Alberta } & No involvement & 12,591 & 1563 (1490: 1640) \\
\hline & Low involvement & 10,869 & 1383 (1331: 1439) \\
\hline & Medium-high involvement & 6102 & 923 (882: 966) \\
\hline \multirow[t]{3}{*}{ British Columbia } & No involvement & 16,333 & 1400 (1321: 1484) \\
\hline & Low involvement & 16,103 & 1224 (1170: 1281) \\
\hline & Medium-high involvement & 8847 & 774 (738: 812) \\
\hline \multirow[t]{3}{*}{ Manitoba } & No involvement & 4776 & 1288 (1180: 1405) \\
\hline & Low involvement & 5654 & 1247 (1152: 1349) \\
\hline & Medium-high involvement & 4814 & 772 (700: 852) \\
\hline \multirow[t]{3}{*}{ New Brunswick } & No involvement & 3435 & 1324 (1173: 1493) \\
\hline & Low involvement & 3920 & 1007 (919: 1104) \\
\hline & Medium-high involvement & 3637 & 710 (657: 768) \\
\hline \multirow[t]{3}{*}{ Newfoundland } & No involvement & 2499 & 1447 (1318: 1590) \\
\hline & Low involvement & 2388 & 1206 (1120: 1299) \\
\hline & Medium-high involvement & 2312 & 826 (782: 873) \\
\hline \multirow[t]{3}{*}{ Nova Scotia } & No involvement & 3271 & 1386 (1234: 1557) \\
\hline & Low involvement & 4936 & 1248 (1141: 1366) \\
\hline & Medium-high involvement & 5287 & 755 (693: 823) \\
\hline \multirow[t]{3}{*}{ Ontario } & No involvement & 46,639 & 1394 (1336: 1454) \\
\hline & Low involvement & 46,200 & 1181 (1139: 1225) \\
\hline & Medium-high involvement & 25,571 & 726 (701: 752) \\
\hline \multirow[t]{3}{*}{ PEl } & No involvement & 675 & 1694 (1268: 2264) \\
\hline & Low involvement & 696 & 1420 (1082: 1863) \\
\hline & Medium-high involvement & 485 & 1029 (779: 1359) \\
\hline \multirow[t]{3}{*}{ Saskatchewan } & No involvement & 5231 & 1537 (1435: 1646) \\
\hline & Low involvement & 4083 & 1383 (1328: 1439) \\
\hline & Medium-high involvement & 3286 & 898 (852: 947) \\
\hline
\end{tabular}

${ }^{\text {a }}$ Model adjusted for age, sex, van Walraven index

had the lowest percentage of people hospitalized primarily for palliative care (19\%) [19]. Despite these variations, we observed that the dose-response relationship was maintained across provinces.

Our paper has several limitations. First, costs were derived in a top-down approach using resource intensity weights, which is less precise than a microcosting approach; thereby limiting the generalizability of our findings. Second, there may be inconsistencies with how often and under what circumstances palliative care is coded across the provinces. Challenges with the subjective nature of the deeming palliative care as the most responsible diagnosis code and the risk of this designation being overused or misused was noted by Downar et al. in 2010 [36]; however, these issues persist. Third, the method we used for identifying receipt of palliative care, approximates receipt of palliative care, but further research is needed to verify that these methods are truly identifying encounters with palliative care providers and that the designation of low or medium/high is appropriate. This method also does not capture the intensity of palliative care receipt. Fourth, there are two main limitations with using a decedent cohort in terminal hospitalizations. We cannot draw causal inferences; however, we can examine the costs of care provided at the end of life. Further physicians do not necessarily know when a patient is admitted to hospital that he/she will die in hospital, so our findings cannot necessarily be leveraged for prospective decision making. While prognostication is inexact, future prospective studies may more precisely be able to examine the relationship between palliative care involvement, hospitalizations, and costs. Fifth, there are limitations to using the DAD; we are unable to measure the frequency of palliative care (i.e., how many 
Table 5 Estimated costs per day and 95\% confidence intervals from adjusted ${ }^{a}$ gamma Generalized Estimating Equation model, stratified by Elixhauser disease condition

\begin{tabular}{|c|c|c|c|}
\hline Disease & Palliative Care Intensity & $\mathrm{n}$ & Cost $(95 \% \mathrm{Cl})$ \\
\hline \multirow[t]{3}{*}{ Metastatic cancer } & No involvement & 6992 & 1204 (1172: 1237) \\
\hline & Low involvement & 19,475 & 1038 (1021: 1055) \\
\hline & Medium-high involvement & 26,267 & 754 (746: 762) \\
\hline \multirow[t]{3}{*}{ Heart failure } & No involvement & 25,076 & 1275 (1248: 1303) \\
\hline & Low involvement & 21,711 & 1178 (1158: 1198) \\
\hline & Medium-high involvement & 5723 & 814 (796: 832) \\
\hline \multirow[t]{3}{*}{ Major neurocognitive disorders } & No involvement & 7393 & 1578 (1530: 1628) \\
\hline & Low involvement & 8545 & 1448 (1408: 1489) \\
\hline & Medium-high involvement & 3311 & 835 (811: 859) \\
\hline \multirow[t]{3}{*}{ COPD } & No involvement & 17,240 & 1298 (1273: 1323) \\
\hline & Low involvement & 16,541 & 1246 (1223: 1269) \\
\hline & Medium-high involvement & 5950 & 789 (774: 804) \\
\hline \multirow[t]{3}{*}{ Kidney failure } & No involvement & 10,042 & 1307 (1278: 1336) \\
\hline & Low involvement & 9749 & 1201 (1179: 1224) \\
\hline & Medium-high involvement & 3339 & 829 (810: 849) \\
\hline \multirow[t]{3}{*}{ HIV } & No involvement & 181 & 1917 (1791: 2052) \\
\hline & Low involvement & 170 & 1817 (1684: 1961) \\
\hline & Medium-high involvement & 67 & 973 (857: 1104) \\
\hline \multirow[t]{3}{*}{ Liver Failure } & No involvement & 6295 & 1668 (1616: 1723) \\
\hline & Low involvement & 7457 & 1459 (1417: 1502) \\
\hline & Medium-high involvement & 2344 & 796 (768: 826) \\
\hline
\end{tabular}

${ }^{a}$ Model adjusted for age, sex, province, van Walraven index

times a patient was seen by palliative care specialists) or the quality of palliative care, when in the hospitalization palliative care services were received, what constitutes a palliative care service or provider, and concordance between preferences for care and services received. While in our adjusted models we controlled for variables we thought might confound the relationship between palliative care and costs, there may still be uncontrolled confounding regarding who receives palliative care, as well as the availability of hospital-based, specialist palliative care. Finally, our data was restricted to inpatient data, which precluded us from assessing the impact that outpatient palliative care might have on our results. Outpatient palliative care has been associated with decreased health utilization and increased likelihood of home deaths [3741], which might lead patients receiving outpatient palliative care to be excluded from our cohort.

\section{Conclusions}

Increased palliative care involvement is associated with lower inpatient costs. This is the first national population-representative analysis on the impact of inpatient palliative care on costs using a tiered palliative care variable. Future research should explore whether this relationship holds for other hospitalizations towards the end of life, as well as whether palliative care in other settings impacts inpatient costs.

\section{Abbreviations}

AB: Alberta; BC: British Columbia; Cl: Confidence intervals; COPD: Chronic obstructive pulmonary disease; CPI : Consumer Price Index;

CPR: Cardiopulmonary resuscitation; DAD: Discharge Abstract Database; F: Female; GEE: Generalized estimating equation; HIV/AIDS: Human immunodeficiency virus/acquired immunodeficiency syndrome;

ICD: International Statistical Classification of Diseases and Related Health Problems; ICU: Intensive care unit; IQR: Interquartile range; MB: Manitoba; NB : New Brunswick; NL: Newfoundland; NS: Nova Scotia; ON: Ontario; PE: Prince Edward Island; SD: Standard deviation; SK: Saskatchewan

\section{Supplementary Information}

The online version contains supplementary material available at https://doi. org/10.1186/s12913-021-06335-1.

Additional file 1: Figure 1. Cohort creation figure. Table 1. Median/ IQR cost per day in each level of palliative involvement and hospital length of stay.

\section{Acknowledgements}

At the time that this work was completed, Sarina R. Isenberg was an employee at the Temmy Latner Centre for Palliative Care at Sinai Health in Toronto, Ontario.

This work was previously presented at the virtual American Association for Hospice Palliative Medicine Conference in January 2021. Consequently, a 
version of the abstract of this manuscript was published in the Journal of Pain and Symptom Management: Isenberg, Sarina R., et al. "The Association Between Varying Levels of Palliative Care Involvement on Costs During Terminal Hospitalizations in Canada from 2012-2015 (F405C)." Journal of Pain and Symptom Management 61.3 (2021): 649.

\section{Authors' contributions}

SRI conceived of the study, designed the study, supported analysis and interpretation of the data, and drafted the manuscript. CM designed the study, led analysis, supported writing of the manuscript. PM designed the study, supported analysis and interpretation of the data. PT conceived of the study, supported analysis and interpretation of the data. KQ supported analysis and interpretation of the data. DQ supported analysis and interpretation of the data. SS supported analysis and interpretation of the data. CW designed the study, supported analysis and interpretation of the data. HS conceived of the study, supported analysis and interpretation of the data. JD supported analysis and interpretation of the data. TJS supported analysis and interpretation of the data. AH supported analysis and interpretation of the data. PGL supported analysis and interpretation of the data. RF supported analysis and interpretation of the data. JL supported analysis and interpretation of the data. KM conceived of the study, designed the study, and supported analysis and interpretation of the data. ATH conceived of the study, designed the study, supported analysis and interpretation of the data, and supported writing of the manuscript. All authors read and approved the final manuscript.

\section{Authors' information}

Not applicable.

\section{Funding}

This study was funded by a research grant from the Canadian Institutes of Health Research (CIHR SDA-156943). Dr. May's time was supported by the Health Research Board in Ireland (ARPP-A-2018-005). Parts of this material are based on data and information compiled and provided by the Canadian Institute for Health Information. However, the analyses, conclusions, opinions and statements expressed herein are those of the authors, and not necessarily those of Canadian Institute for Health Information.

\section{Availability of data and materials}

The data that support the findings of this study are available from Canadian Institute for Health Information, but restrictions apply to the availability of these data, which were used under license for the current study, and so are not publicly available.

\section{Declarations}

\section{Ethics approval and consent to participate}

This study received ethics approval from Mount Sinai Hospital (18-0285-C) and the Ottawa Hospital/Ottawa Hospital Research Institute (20180719-01H). As this study was analysis of administrative data that is routinely collected by the Ontario government, we did not obtain written nor verbal consent from participants. To access the Discharge Abstract Database data, we completed the necessary privacy protocols with the Canadian Institute for Health Information as well as paid fees for an analyst to provide a cut of the data.

\section{Consent for publication}

Not applicable.

\section{Competing interests}

The authors declare that they have no competing interests.

\section{Author details}

${ }^{1}$ Bruyère Research Institute, 43 Bruyère St, Office 264J-G, Ottawa, ON K1N 5C8, Canada. ${ }^{2}$ Department of Family and Community Medicine, University of Toronto, Toronto, Canada. ${ }^{3}$ Institute for Health Policy Management and Evaluation, University of Toronto, Toronto, Canada. ${ }^{4}$ Department of Medicine, University of Ottawa, Ottawa, Canada. ${ }^{5}$ Centre for Health Policy and Management, Trinity College Dublin, Dublin, Ireland. ${ }^{6}$ The Irish Longitudinal study on Ageing (TILDA), Trinity College Dublin, Dublin, Ireland. ${ }^{7}$ Division of Palliative Care, Department of Medicine, University of Ottawa, Ottawa, Canada. ${ }^{8}$ Ottawa Hospital Research Institute, Ottawa, Canada. ${ }^{9}$ Department of
Medicine, University of Toronto, Toronto, Canada. ${ }^{10}$ Department of Medicine, Division of Internal Medicine, Sinai Health, Toronto, Canada. ${ }^{11}$ Temmy Latner Centre for Palliative Care, Sinai Health, Toronto, Canada. ${ }^{12}$ Department of Oncology, McMaster University, Hamilton, Canada. ${ }^{13}$ Department of Medicine, Johns Hopkins Hospital and Health System, Baltimore, USA. ${ }^{14}$ Department of Oncology, Johns Hopkins Hospital and Health System, Baltimore, USA. ${ }^{15}$ Interdepartmental Division of Critical Care Medicine, University of Toronto, Toronto, Canada. ${ }^{16}$ Tory Trauma Program, Sunnybrook Hospital, Toronto, Canada. ${ }^{17}$ End-of-Life Care Unit, Strategic Policy Branch, Health Canada, Ottawa, Canada. ${ }^{18}$ Centre for Health Services and Policy Research, School of Population and Public Health, The University of British Columbia, Vancouver, Canada.

Received: 26 July 2020 Accepted: 30 March 2021

Published online: 13 April 2021

\section{References}

1. Bekelman JE, Halpern SD, Blankart CR, Bynum JP, Cohen J, Fowler R, et al. Comparison of site of death, health care utilization, and hospital expenditures for patients dying with cancer in 7 developed countries. JAMA. 2016;315(3):272-83. https://doi.org/10.1001/jama.2015.18603.

2. Statistics Canada. Table 13-10-0715-01 Deaths, by place of death (hospital or non-hospital) 2018. doi: https://doi.org/10.25318/1310071501-eng.

3. Tanuseputro $P$, Wodchis WP, Fowler R, Walker $\mathrm{P}$, Bai $Y Q$, Bronskill SE, et al. The health care cost of dying: a population-based retrospective cohort study of the last year of life in Ontario, Canada. PLoS One. 2015;10(3): e0121759. https://doi.org/10.1371/journal.pone.0121759.

4. Hill AD, Stukel TA, Fu L, Scales DC, Laupacis A, Rubenfeld GD, et al. Trends in site of death and health care utilization at the end of life: a populationbased cohort study. CMAJ Open. 2019;7(2):E306-E15. https://doi.org/10. 9778/cmajo.20180097.

5. May P, Normand C, Cassel JB, Del Fabbro E, Fine RL, Menz R, et al. Economics of palliative care for hospitalized adults with serious illness: a meta-analysis. JAMA Intern Med. 2018;178(6):820-9. https://doi.org/10.1001/ jamainternmed.2018.0750.

6. McCarthy IM, Robinson C, Huq S, Philastre M, Fine RL. Cost savings from palliative care teams and guidance for a financially viable palliative care program. Health Serv Res. 2015;50(1):217-36. https://doi.org/10.1111/14756773.12203.

7. O'Connor NR, Junker P, Appel SM, Stetson RL, Rohrbach J, Meghani SH. Palliative care consultation for goals of care and future acute care costs: a propensity-matched study. Am J Hosp Palliat Care. 2018;35(7):966-71. https://doi.org/10.1177/1049909117743475.

8. Penrod JD, Deb P, Luhrs C, Dellenbaugh C, Zhu CW, Hochman T, et al. Cost and utilization outcomes of patients receiving hospital-based palliative care consultation. J Palliat Med. 2006;9(4):855-60. https://doi.org/10.1089/jpm.2 006.9.855.

9. Simoens S, Kutten B, Keirse E, Berghe PV, Beguin C, Desmedt M, et al. Costs of terminal patients who receive palliative care or usual care in different hospital wards. J Palliat Med. 2010;13(11):1365-9. https://doi.org/10.1089/ jpm.2010.0212.

10. Elixhauser A, Steiner C, Harris DR, Coffey RM. Comorbidity measures for use with administrative data. Med Care. 1998;36(1):8-27. https://doi.org/10.1097/ 00005650-199801000-00004.

11. van Walraven C, Austin PC, Jennings A, Quan H, Forster AJ. A modification of the Elixhauser comorbidity measures into a point system for hospital death using administrative data. Med Care. 2009;47(6):626-33. https://doi. org/10.1097/MLR.0b013e31819432e5.

12. Siddiqui NF, Coca SG, Devereaux PJ, Jain AK, Li L, Luo J, et al. Secular trends in acute dialysis after elective major surgery--1995 to 2009. CMAJ. 2012; 184(11):1237-45. https://doi.org/10.1503/cmaj.110895.

13. Webber C, Chan R, Scott M, Brown C, Spruin S, Hsu AT, et al. The delivery of palliative care in acute care hospitals: a population-based retrospective cohort study describing the level of involvement and timing of inpatient palliative care in the last year of life. Palliat Med. 2020; In press.

14. Wodchis WP, Bushmeneva K, Nikitovic M, McKillop I. Guidelines on person level cost using administrative databases in Ontario. Toronto: Health System Performance Research Network (HSPRN); 2013.

15. Jacobs P, Budden A, Lee KM. Guidance document for the costing of health care resources in the Canadian setting. Ottawa: CADTH; 2016. 
16. Glussich A. Estimating costs of a hospital stay. Available from: https://www cadth.ca/sites/default/files/symp-2016/presentations/april12-2016/ Concurrent-Session-E3-Anyk-Glussich.pptx. Accessed 15 Jan 2020.

17. Canadian Institute for Health Information. Canadian patient cost database: technical document. Ottawa: $\mathrm{ClH} ; 2019$.

18. Statistics Canada. Table 18-10-0005-01 Consumer Price Index, annual average, not seasonally adjusted. Available from: https://www150.statcan.gc. ca/t1/tbl1/en/tv.action?pid=1810000501. Accessed 15 Jan 2020.

19. Canadian Institute for Health Information. Access to palliative care in Canada. Ottawa: $\mathrm{ClHl} ; 2018$

20. May P, Normand C, Del Fabbro E, Fine RL, Morrison RS, Ottewill I, et al Economic analysis of hospital palliative care: investigating heterogeneity by noncancer diagnoses. MDM Policy Pract. 2019;4(2):2381468319866451. https://doi.org/10.1177/2381468319866451.

21. Hsu A, Garner R. Associations between the receipt of inpatient palliative care and acute care outcomes: a restrospective study. Health Rep. 2020; 31(10):3-13. https://doi.org/10.25318/82-003-x202001000001-eng.

22. Seow H, Qureshi D, Isenberg SR, Tanuseputro P. Access to palliative care during a terminal hospitalization. J Palliat Med. 2020;23(12):1644-8.

23. May P, Normand C, Morrison RS. Economic impact of hospital inpatient palliative care consultation: review of current evidence and directions for future research. J Palliat Med. 2014;17(9):1054-63. https://doi.org/10.1089/ jpm.2013.0594.

24. O'Mahony S, Blank AE, Zallman L, Selwyn PA. The benefits of a hospital-based inpatient palliative care consultation service: preliminary outcome data. J Palliat Med. 2005;8(5):1033-9. https://doi.org/10.1089/jpm.2005.8.1033.

25. May P, Garrido MM, Cassel JB, Kelley AS, Meier DE, Normand C, et al. Cost analysis of a prospective multi-site cohort study of palliative care consultation teams for adults with advanced cancer: where do cost-savings come from? Palliat Med. 2017;31(4):378-86. https://doi.org/10.1177/026921 6317690098.

26. Lin WY, Chiu TY, Ho CT, Davidson LE, Hsu HS, Liu CS, et al. Hospice sharedcare saved medical expenditure and reduced the likelihood of intensive medical utilization among advanced cancer patients in Taiwan--a nationwide survey. Support Care Cancer. 2014;22(7):1907-14. https://doi. org/10.1007/s00520-014-2168-5

27. Vogl M, Schildmann E, Leidl R, Hodiamont F, Kalies H, Maier BO, et al. Redefining diagnosis-related groups (DRGs) for palliative care - a crosssectional study in two German centres. BMC Palliat Care. 2018;17(1):58. https://doi.org/10.1186/s12904-018-0307-3.

28. Nathanson BH, McGee WT, Dietzen DL, Chen Q, Young J, Higgins TL. A state-level assessment of hospital-based palliative care and the use of lifesustaining therapies in the United States. J Palliat Med. 2016;19(4):421-7. https://doi.org/10.1089/jpm.2015.0233.

29. Khandelwal N, Kross EK, Engelberg RA, Coe NB, Long AC, Curtis JR. Estimating the effect of palliative care interventions and advance care planning on ICU utilization. Crit Care Med. 2015;43(5):1102-11. https://doi. org/10.1097/CCM.0000000000000852.

30. Macmillan PJ, Chalfin B, Soleimani Fard A, Hughes S. Earlier palliative care referrals associated with reduced length of stay and hospital charges. J Palliat Med. 2019;23(1):107-11.

31. Jang RW, Krzyzanowska MK, Zimmermann C, Taback N, Alibhai SM. Palliative care and the aggressiveness of end-of-life care in patients with advanced pancreatic cancer. J Natl Cancer Inst. 2015;107(3):dju424.

32. Hua M, Wunsch $\mathrm{H}$. Integrating palliative care in the ICU. Curr Opin Crit Care. 2014;20(6):673-80. https://doi.org/10.1097/MCC.0000000000000149.

33. Rolnick JA, Ersek M, Wachterman MW, Halpern SD. The quality of end-of-life care among intensive care unit versus ward decedents. Am J Respir Crit Care Med. 2020;201(7):832-9.

34. Doyle CM, Lix LM, Hemmelgarn BR, Paterson JM, Renoux C. Data variability across Canadian administrative health databases: differences in content, coding, and completeness. Pharmacoepidemiol Drug Saf. 2020;29(S1):68-77. https://doi.org/10.1002/pds.4889.

35. Canadian Institute for Health Information. National health expenditure trends, 1975 to 2019. Ottawa: ClHI; 2019

36. Downar J, Sibbald R, Lazar NM. Ethical considerations for classifying patients as 'palliative' when calculating hospital standardised mortality ratios. J Med Ethics. 2010;36(7):387-90. https://doi.org/10.1136/jme.2010.035451.

37. Kraskovsky V, Schneider J, Mador MJ, Provost KA. Longer duration of palliative care in patients with COPD is associated with death outside the hospital. J Palliat Care. 2019:825859719851486. Online ahead of print.
38. Gomes B, Calanzani N, Curiale V, Mccrone P, lj H. Effectiveness and costeffectiveness of home palliative care services for adults with advanced illness and their caregivers ( review ). Cochrane Rev. 2014;(6):CD007760.

39. Davis MP, Temel JS, Balboni T, Glare P. A review of the trials which examine early integration of outpatient and home palliative care for patients with serious illnesses. Ann Palliative Med. 2015;4(3):99-121. https://doi.org/10.3 978/j.issn.2224-5820.2015.04.04

40. Rabow M, Kvale E, Barbour L, Cassel JB, Cohen S, Jackson V, et al. Moving upstream: a review of the evidence of the impact of outpatient palliative care. J Palliat Med. 2013;16(12):1540-9. https://doi.org/10.1089/.jpm.2013.0153.

41. Seow H, Brazil K, Barbera L, Sussman J, Pereira J, Marshall D, et al. The effectiveness of palliative care expert-consult teams: focus on the Temmy Latner Centre for Palliative Care (TLCPC) Team, Toronto ON. 2013.

\section{Publisher's Note}

Springer Nature remains neutral with regard to jurisdictional claims in published maps and institutional affiliations.
Ready to submit your research? Choose BMC and benefit from:

- fast, convenient online submission

- thorough peer review by experienced researchers in your field

- rapid publication on acceptance

- support for research data, including large and complex data types

- gold Open Access which fosters wider collaboration and increased citations

- maximum visibility for your research: over $100 \mathrm{M}$ website views per year

At $\mathrm{BMC}$, research is always in progress.

Learn more biomedcentral.com/submissions 